\title{
ASYMPTOTIC SPEED OF PROPAGATION FOR A VISCOUS SEMILINEAR PARABOLIC EQUATION
}

\author{
Annalisa Cesaroni $^{1}$, Nicolas Dirr $^{2}$ and Matteo Novaga ${ }^{3}$
}

\begin{abstract}
We characterize the asymptotic speed of propagation of almost planar solutions to a semilinear viscous parabolic equation, with periodic nonlinearity.

Résumé. Nous caractérisons la vitesse asymptotique de propagation des solutions presque planaires d'une équation parabolique visqueuse semilinéaire, avec une non-linéarité périodique.
\end{abstract}

\section{INTRODUCTION}

We are interested in the asymptotic behavior, as $\varepsilon \rightarrow 0$, of solutions to the following problem

$$
u_{t}^{\varepsilon}-\varepsilon^{\alpha} \Delta u^{\varepsilon}+g\left(\frac{u^{\varepsilon}}{\varepsilon}\right)=0 \quad x \in \mathbb{R}^{n}, t>0
$$

where $\alpha \in[0,1)$ and $g: \mathbb{R} \rightarrow \mathbb{R}$ is a Lipschitz continuous, 1-periodic function, with $g(v) \leq 0$.

This equation can be interpreted as a simplified model for the motion of an interface in a material under the combined effect of a spatially heterogeneous medium, given by $g$, and the surface tension. Note that the Laplacian is the linearized version of the curvature operator and the interface is given by the graph of the function $u$.

If we perform the parabolic rescaling

$$
v^{\varepsilon}(x, t)=\frac{1}{\varepsilon} u^{\varepsilon}\left(\varepsilon x, \varepsilon^{2-\alpha} t\right)
$$

equation (1) becomes

$$
v_{t}^{\varepsilon}-\Delta v^{\varepsilon}+\varepsilon^{1-\alpha} g\left(v^{\varepsilon}\right)=0 .
$$

Therefore, the analysis of the limit as $\varepsilon \rightarrow 0$ of solutions to (1) is related to the long time behavior of solutions of (2).

When $\varepsilon=1$ or equivalently $\alpha=1$, the long time behavior has been considered in the literature by several authors, also in the case the Laplacian is substituted by a more general elliptic operator, and the existence of special solutions such as traveling or pulsating waves has been established (see $[2-5,7,9,10,14,15]$ and references therein).

\footnotetext{
1 Dipartimento di Scienze Statistiche Università di Padova Via Cesare Battisti 241/243, 35121 Padova, Italy

2 Cardiff School of Mathematics Cardiff University, Senghennydd Road, Cardiff, Wales, UK

3 Dipartimento di Matematica Università di Pisa, Largo Bruno Pontecorvo 5, 56127 Pisa, Italy
} 
In $[1,12]$ it has been studied the limit behavior of solutions of

$$
u_{t}^{\varepsilon}-\Delta u^{\varepsilon}+\frac{1}{\varepsilon} g\left(\frac{u^{\varepsilon}}{\varepsilon}\right)=0 \quad x \in \mathbb{R}^{n}, t>0,
$$

under the assumption that $\int_{0}^{1} g=0$. Note that this equation can be obtained from (1) with $\alpha=1$ under a time rescaling.

In this note we are interested in the case $\alpha \in[0,1)$. The main result is Theorem 3.1 which provides the effective speed of propagation of solutions to (1) starting from almost planar initial datum. In particular we show that the solution to (1) with initial datum $u^{\varepsilon}(x, 0)=p \cdot x+k$ satisfies

$$
\lim _{\varepsilon \rightarrow 0} u^{\varepsilon}(x, t)=p \cdot x+k+c(p) t \quad \text { uniformly in } x, t
$$

where the average positive speed $c(p)$ depends on $g$.

The proof of this result relies on maximum principle arguments and on a priori bounds on the gradient of solutions to (1) with planar initial data. These bounds are provided by Theorem 1.1 and are based on the so called Bernstein method (see $[9,13,14]$ ).

The interesting feature we observe is that in the case $\alpha<1$, the asymptotic speed of propagation $c(p)$ is just lower semicontinuous, but not continuous with respect to $p$, whereas when $\alpha=1$, the speed $c(p)$ depends continuously on $p$, as it has been proved in [5,9]. In particular, we show in Proposition 2.2 that

$$
c(p)= \begin{cases}-\int_{0}^{1} g(s) d s & p \neq 0 \\ -\left(\int_{0}^{1} g^{-1}(s) d s\right)^{-1} & p=0\end{cases}
$$

so that the limit speed is discontinuous with respect to the slope $p$. Such phenomenon is unusual in homogenization problems, and indicates that the effective limit of (1) as $\varepsilon \rightarrow 0$ is governed by a differential operator which is discontinuous in the gradient entry. An equation similar to (1), with $n=1$ and $\alpha=0$, giving rise to a discontinuous effective limit problem, has been considered in [8].

This makes the analysis of this limit more challenging, and will be the topic of a paper in preparation [6], where we will consider the long time behaviour and the asymptotic limit of solutions to

$$
u_{t}^{\varepsilon}-\varepsilon^{\alpha} \Delta u^{\varepsilon}+g\left(\frac{x}{\varepsilon}, \frac{u^{\varepsilon}}{\varepsilon}\right)=0 \quad x \in \mathbb{R}^{n}, t>0
$$

where $g: \mathbb{R}^{n} \times \mathbb{R} \rightarrow \mathbb{R}$ is Lipschitz continuous and $\mathbb{Z}^{n+1}$-periodic.

Acknowledgments. The authors would like to thank the mathematics departments of the Universities of Padova, Firenze and Pisa for the kind hospitality during the preparation of this work. A.C. was partially supported by the GNAMPA Project 2015 "Processi di diffusione degeneri o singolari legati al controllo di dinamiche stocastiche". N.D was partially supported by the University of Padova through the Visiting Scientist Programme, and by the Leverhulme Trust through RPG-2013-261. M.N. was partially supported by the University of Pisa via Grant PRA-2015-0017.

\section{A priori EStimates ON THE GRADIENT}

We consider in this section the more general case in which $g: \mathbb{R}^{n} \times \mathbb{R} \rightarrow \mathbb{R}$ is a Lipschitz, $\mathbb{Z}^{n+1}$ periodic function.

We introduce, for any $p \in \mathbb{R}^{n}$, the initial value problem

$$
\left\{\begin{array}{l}
v_{t}^{\varepsilon}-\Delta v^{\varepsilon}+\varepsilon^{1-\alpha} g\left(x, v^{\varepsilon}\right)=0 \\
v^{\varepsilon}(x, 0)=p \cdot x
\end{array}\right.
$$


We recall that this problem admits a unique solution $v^{\varepsilon} \in C^{2+\gamma, 1+\gamma / 2}$ for all $\gamma \in(0,1)$. Moreover we recall also that, due to Lipschitz regularity of $g$, a standard comparison principle among sub and supersolutions to (4) holds (see [16]).

We provide an a priori bound on the oscillation and on the gradient of the solutions to (4), which will be useful in the following. The approach is based on the so called Bernstein type method (see $[9,13,14])$.

In the following we let $w^{\varepsilon}(x, t)=v^{\varepsilon}(x, t)-p \cdot x$, where $v^{\varepsilon}$ is the unique solution to (4). Notice that $w^{\varepsilon}$ solves

$$
\left\{\begin{array}{l}
w_{t}-\Delta w+\varepsilon^{1-\alpha} g(x, w+p \cdot x)=0 \\
w(x, 0)=0
\end{array}\right.
$$

Theorem 1.1. Given $p \in \mathbb{R}^{n}$, then there exists $\varepsilon_{0}=\varepsilon(|p|)$ such that for every $\varepsilon \leq \varepsilon_{0}$ and every $t \geq 0$ there holds

$$
\sup _{x \in \mathbb{R}^{n}}\left|D w^{\varepsilon}(x, t)\right| \leq C_{n} \varepsilon^{\frac{1-\alpha}{4}}(1+|p|)\|g\|_{1, \infty}
$$

and

$$
\sup _{x \in \mathbb{R}^{n}} w^{\varepsilon}(x, t)-\inf _{x \in \mathbb{R}^{n}} w^{\varepsilon}(x, t) \leq 2+C_{n} \varepsilon^{\frac{1-\alpha}{4}}(1+|p|)\|g\|_{1, \infty}
$$

where $C_{n}$ is a constant depending on the space dimension and $\|g\|_{1, \infty}$ is the Lipschitz norm of $g$.

Proof. The proof of the theorem is based on similar arguments as in [9, Thm 2.4, Cor. 2.5]. We divide the proof in several steps.

Step 1: we prove that for every $t \geq 0$

$$
\operatorname{osc}\left(w^{\varepsilon}(\cdot, t),[0,1]^{n}\right) \leq \operatorname{osc}\left(w^{\varepsilon}(\cdot, t), \mathbb{R}^{n}\right) \leq \operatorname{osc}\left(w^{\varepsilon}(\cdot, t),[0,1]^{n}\right)+2,
$$

where $\operatorname{osc}\left(w^{\varepsilon}(\cdot, t), A\right)=\sup _{x \in A} w^{\varepsilon}(x, t)-\inf _{x \in A} w^{\varepsilon}(x, t)$.

Fix $t>0$ and $\ell \in \mathbb{Z}^{n}$ and let $\gamma=[p \cdot \ell]+1-p \cdot \ell \in(0,1]$. We define $\hat{w}^{\varepsilon}(x, t):=w^{\varepsilon}(x-\ell, t)+\gamma$. Observe that, due to periodicity of $g$, it satisfies the same equation as $w^{\varepsilon}$ :

$$
\hat{w}_{t}^{\varepsilon}(x, t)=\Delta w^{\varepsilon}(x-\ell, t)-\varepsilon^{1-\alpha} g\left(x-\ell, w^{\varepsilon}(x-\ell, t)+p \cdot(x-\ell)\right)=\Delta \hat{w}^{\varepsilon}-\varepsilon^{1-\alpha} g\left(x, \hat{w}^{\varepsilon}+p \cdot x\right) .
$$

Moreover $\hat{w}^{\varepsilon}(x, 0):=w^{\varepsilon}(x-\ell, 0)+\gamma=\gamma>0$. Therefore by the comparison principle, we obtain

$$
w^{\varepsilon}(x, t) \leq w^{\varepsilon}(x-\ell, t)+\gamma \leq w^{\varepsilon}(x-\ell, t)+1 \quad \forall x \in \mathbb{R}^{n}, \ell \in \mathbb{Z}^{n} .
$$

This implies immediately (6).

Step 2: properties of the function $W^{\varepsilon}(t)=\sup _{x \in \mathbb{R}^{n}} w^{\varepsilon}(x, t)$.

For simplicity from now on we consider the case in which $g \in C^{\infty}\left(\mathbb{R}^{n+1}\right)$. The case of $g$ just Lipschitz is recovered by a standard approximation procedure.

Note that, by comparison principle, $0 \leq w^{\varepsilon}(x, t) \leq \varepsilon^{1-\alpha}\|g\|_{\infty} t$, for all $x, t$. Moreover, again by comparison principle,

$$
\inf _{y \in \mathbb{R}^{n}} w^{\varepsilon}(y, s) \leq w^{\varepsilon}(x, t) \leq \sup _{y \in \mathbb{R}^{n}} w^{\varepsilon}(y, s)+\varepsilon^{1-\alpha}(t-s)\|g\|_{\infty} \quad \forall t \geq s .
$$

This implies that the function

$$
W^{\varepsilon}(t)=\sup _{x \in \mathbb{R}^{n}} w^{\varepsilon}(x, t)
$$

satisfies, in viscosity sense,

$$
W_{t}^{\varepsilon} \leq \varepsilon^{1-\alpha}\|g\|_{\infty}
$$

Let $\lambda>0$ to be fixed later. We consider the function

$$
\phi^{\varepsilon}(x, t)=\frac{\left|D w^{\varepsilon}(x, t)\right|^{2}}{2}-\lambda w^{\varepsilon}(x, t)+\lambda W^{\varepsilon}(t) .
$$


Note that $\frac{\left|D w^{\varepsilon}(x, t)\right|^{2}}{2} \leq \phi^{\varepsilon}(x, t) \leq \frac{\left|D w^{\varepsilon}(x, t)\right|^{2}}{2}+\lambda \operatorname{osc}\left(w^{\varepsilon}(\cdot, t), \mathbb{R}^{n}\right)$, and moreover $\phi^{\varepsilon}(x, 0)=0$. Let $\Phi^{\varepsilon}(t)=$ $\sup _{x \in \mathbb{R}^{n}} \phi^{\varepsilon}(x, t)$.

Step 3: we prove that choosing $\lambda=C \varepsilon^{(1-\alpha) / 2} n^{1 / 2}(1+|p|)\left(\|D g\|_{\infty}+\|g\|_{\infty}\right)$, for $C>1$, then for every $\varepsilon$ sufficiently small

$$
\Phi^{\varepsilon}(t) \leq \lambda \sup _{t \in[0, T]} \operatorname{osc}\left(w^{\varepsilon}(\cdot, t), \mathbb{R}^{n}\right)
$$

for every $t \in[0, T]$, where $T>0$ is the stopping time

$$
T=\inf \left\{s \geq 0: \sup _{x}\left|D w^{\varepsilon}(x, s)\right|=1\right\}
$$

Let $t^{*}$ such that $\Phi^{\varepsilon}\left(t^{*}\right) \geq \Phi^{\varepsilon}(t)$ for every $t \in[0, T]$. If $t^{*}=0$, then $\Phi^{\varepsilon}(t) \leq 0$ for every $t$ and we are done. Assume that $t^{*}>0$. It is easy to show that there exists a sequence $x_{k}\left(t^{*}\right)$ such that

$$
\phi^{\varepsilon}\left(x_{k}\left(t^{*}\right), t^{*}\right) \rightarrow \Phi^{\varepsilon}\left(t^{*}\right)
$$

We can choose the sequence such that

$$
D \phi^{\varepsilon}\left(x_{k}\left(t^{*}\right), t^{*}\right) \rightarrow 0 \quad \lim _{k} \Delta \phi^{\varepsilon}\left(x_{k}\left(t^{*}\right), t^{*}\right) \leq 0 .
$$

Finally,

$$
\lim _{k} \phi_{t}^{\varepsilon}\left(x_{k}\left(t^{*}\right), t^{*}\right) \geq 0
$$

We claim that, choosing appropriately $\lambda, \lim _{k}\left|D w^{\varepsilon}\left(x_{k}\left(t^{*}\right), t^{*}\right)\right|=0$. If it is true, then

$$
\Phi^{\varepsilon}(t) \leq \Phi^{\varepsilon}\left(t^{*}\right) \leq \lambda \operatorname{osc}\left(w^{\varepsilon}\left(\cdot, t^{*}\right), \mathbb{R}^{n}\right) \leq \lambda \sup _{t \in[0, T]} \operatorname{osc}\left(w^{\varepsilon}(\cdot, t), \mathbb{R}^{n}\right),
$$

and then we get the desired conclusion.

Assume by contradiction that the claim is not true. Then, for every $\lambda$, there exists a subsequence such that $\lim _{k}\left|D w^{\varepsilon}\left(x_{k}\left(t^{*}\right), t^{*}\right)\right|>0$. We compute

$$
\left\{\begin{array}{l}
\phi_{x_{i}}^{\varepsilon}=\sum_{j} w_{x_{i}, x_{j}}^{\varepsilon} w_{x_{j}}^{\varepsilon}-\lambda w_{x_{i}}^{\varepsilon} \\
\phi_{t}^{\varepsilon}=\sum_{j} w_{x_{j}, t}^{\varepsilon} w_{x_{j}}^{\varepsilon}-\lambda w_{t}^{\varepsilon}+\lambda W_{t}^{\varepsilon} \\
\phi_{x_{i}, x_{i}}^{\varepsilon}=\sum_{j} w_{x_{i}, x_{i}, x_{j}}^{\varepsilon} w_{x_{j}}^{\varepsilon}+\sum_{j}\left(w_{x_{i}, x_{j}}^{\varepsilon}\right)^{2}-\lambda w_{x_{i}, x_{i}}^{\varepsilon}
\end{array}\right.
$$

We recall that $w^{\varepsilon}$ solves

$$
w_{t}^{\varepsilon}-\Delta w^{\varepsilon}+\varepsilon^{1-\alpha} g\left(x, w^{\varepsilon}+p \cdot x\right)=0 .
$$

Differentiating the previous equation with respect to $x_{j}$ we get

$$
w_{x_{j}, t}^{\varepsilon}=\sum_{i} w_{x_{i}, x_{i}, x_{j}}^{\varepsilon}-\varepsilon^{1-\alpha} g_{x_{j}}\left(x, w^{\varepsilon}+p \cdot x\right)-\varepsilon^{1-\alpha} g_{v}\left(x, w^{\varepsilon}+p \cdot x\right)\left(w_{x_{j}}^{\varepsilon}+p_{j}\right)
$$


So, using this equation, (8), (9) and (7), we get

$$
\begin{aligned}
\phi_{t}^{\varepsilon} & =\sum_{j} \sum_{i} w_{x_{i}, x_{i}, x_{j}}^{\varepsilon} w_{x_{j}}^{\varepsilon}-\varepsilon^{1-\alpha} \sum_{j} w_{x_{j}}^{\varepsilon}\left(g_{x_{j}}+p_{j} g_{v}\right)-\varepsilon^{1-\alpha} \sum_{j} g_{v}\left(w_{x_{j}}^{\varepsilon}\right)^{2}-\lambda w_{t}^{\varepsilon}+\lambda W_{t}^{\varepsilon} \\
& =\Delta \phi^{\varepsilon}-\sum_{i} \sum_{j}\left(w_{x_{i}, x_{j}}^{\varepsilon}\right)^{2}+\lambda \Delta w^{\varepsilon}-\varepsilon^{1-\alpha} \sum_{j} w_{x_{j}}^{\varepsilon}\left(g_{x_{j}}+p_{j} g_{v}\right)-\varepsilon^{1-\alpha} \sum_{j} g_{v}\left(w_{x_{j}}^{\varepsilon}\right)^{2}-\lambda w_{t}^{\varepsilon}+\lambda W_{t}^{\varepsilon} \\
& =\Delta \phi^{\varepsilon}-\sum_{i} \sum_{j}\left(w_{x_{i}, x_{j}}^{\varepsilon}\right)^{2}+\varepsilon^{1-\alpha}\left[\lambda g-\sum_{j} w_{x_{j}}^{\varepsilon}\left(g_{x_{j}}+p_{j} g_{v}\right)-\sum_{j} g_{v}\left(w_{x_{j}}^{\varepsilon}\right)^{2}\right]+\lambda W_{t}^{\varepsilon} \\
& \leq \Delta \phi^{\varepsilon}-\sum_{i} \sum_{j}\left(w_{x_{i}, x_{j}}^{\varepsilon}\right)^{2}+2 \varepsilon^{1-\alpha} \lambda\|g\|_{\infty}+\varepsilon^{1-\alpha}\|D g\|_{\infty}(1+|p|)\left(\sum_{j}\left|w_{x_{j}}^{\varepsilon}\right|+\sum_{j}\left|w_{x_{j}}^{\varepsilon}\right|^{2}\right) \\
& \leq \Delta \phi^{\varepsilon}-\sum_{i} \sum_{j}\left(w_{x_{i}, x_{j}}^{\varepsilon}\right)^{2}+2 \varepsilon^{1-\alpha} \lambda\|g\|_{\infty}+\sqrt{n} \varepsilon^{1-\alpha}\|D g\|_{\infty}(1+|p|)\left(\left|D w^{\varepsilon}\right|+\left|D w^{\varepsilon}\right|^{2}\right) .
\end{aligned}
$$

We multiply the first equation in (8) by $w_{x_{i}}^{\varepsilon}$ and compute at $x_{k}\left(t^{*}\right)$ : we have that

$$
\lim _{k} \sum_{i} \sum_{j} w_{x_{i}, x_{j}}^{\varepsilon}\left(x_{k}\left(t^{*}\right)\right) w_{x_{j}}^{\varepsilon}\left(x_{k}\left(t^{*}\right)\right) w_{x_{i}}^{\varepsilon}\left(x_{k}\left(t^{*}\right)\right)-\lambda\left|D w^{\varepsilon}\left(x_{k}\left(t^{*}\right)\right)\right|^{2}=0 .
$$

By Cauchy-Schwartz inequality

$$
\sum_{i} \sum_{j} w_{x_{i}, x_{j}}^{\varepsilon} w_{x_{j}}^{\varepsilon} w_{x_{i}}^{\varepsilon} \leq\left|D w^{\varepsilon}\right| \sum_{i}\left|w_{x_{i}}^{\varepsilon}\right|\left[\sum_{j}\left(w_{x_{i}, x_{j}}^{\varepsilon}\right)^{2}\right]^{1 / 2} \leq\left|D w^{\varepsilon}\right|^{2}\left(\sum_{i} \sum_{j}\left(w_{x_{i}, x_{j}}^{\varepsilon}\right)^{2}\right)^{\frac{1}{2}}
$$

This implies, since $\left|D w^{\varepsilon}\left(x_{k}\left(t^{*}\right)\right)\right| \neq 0$, that

$$
\lim _{k} \sum_{i} \sum_{j}\left(w_{x_{i}, x_{j}}^{\varepsilon}\left(x_{k}\left(t^{*}\right)\right)\right)^{2} \geq \lambda^{2}
$$

We compute (10) at $x_{k}\left(t^{*}\right)$, recalling the definition of $T$, of the sequence $x_{k}\left(t^{*}\right)$ and (10) we get

$$
\begin{aligned}
0 & \leq \lim _{k}\left[\phi_{t}^{\varepsilon}\left(x_{k}\left(t^{*}\right), t^{*}\right)-\Delta \phi^{\varepsilon}\left(x_{k}\left(t^{*}\right), t^{*}\right)\right] \\
& \leq-\lambda^{2}+2 \varepsilon^{1-\alpha} \lambda\|g\|_{\infty}+\sqrt{n} \varepsilon^{1-\alpha}\|D g\|_{\infty}(1+|p|) \sup _{t \in[0, T]} \sup _{x}\left(\left|D w^{\varepsilon}\right|^{2}+\left|D w^{\varepsilon}\right|\right) \\
& \leq-\lambda^{2}+2 \varepsilon^{1-\alpha} \lambda\|g\|_{\infty}+\sqrt{n} \varepsilon^{1-\alpha}\|D g\|_{\infty}(1+|p|) .
\end{aligned}
$$

We claim it is possible to choose $\lambda$ so that the right hand side of (11) is strictly negative getting then a contradiction. Indeed if we choose $\lambda=C \varepsilon^{(1-\alpha) / 2} n^{1 / 2}(1+|p|)\left(\|D g\|_{\infty}+\|g\|_{\infty}\right)$, with $C>1$, the claim is true for every $\varepsilon$ sufficiently small.

Step 4: conclusion. 
By the first step, the definition of $\Phi^{\varepsilon}$ and (6), we get that

$$
\begin{aligned}
\sup _{x} \frac{\left|D w^{\varepsilon}(x, t)\right|^{2}}{2} \leq \Phi^{\varepsilon}(t) & \leq \lambda \sup _{t \in[0, T]} \operatorname{osc}\left(w^{\varepsilon}(\cdot, t), \mathbb{R}^{n}\right) \\
& \leq \lambda \sup _{t \in[0, T]}\left(\operatorname{osc}\left(w^{\varepsilon}(\cdot, t),[0,1]^{n}\right)+2\right) \\
& \leq \lambda \sup _{t \in[0, T]} \sup _{x}\left|D w^{\varepsilon}(x, t)\right|+2 \lambda \\
& \leq \sup _{t \in[0, T]} \sup _{x} \frac{\left|D w^{\varepsilon}(x, t)\right|^{2}}{4}+\lambda^{2}+2 \lambda,
\end{aligned}
$$

which in turn gives

$$
\sup _{x}\left|D w^{\varepsilon}(t, x)\right|^{2} \leq 4 \lambda+2 \lambda^{2} \quad \forall t \in[0, T] .
$$

Recall by the first step that $\lambda=C \varepsilon^{(1-\alpha) / 2} n^{1 / 2}(1+|p|)\left(\|D g\|_{\infty}+\|g\|_{\infty}\right)$, so choosing $\varepsilon$ sufficiently small, we obtain $4 \lambda+2 \lambda^{2}<1$. This implies, recalling (12), that the stopping time $T=+\infty$.

Finally the desired estimate on the gradient is obtained by (12), recalling the explicit formula for $\lambda$, and the estimate on the oscillation is deduced by recalling (6).

\section{Existence of ALMOST-PLANAR SOLUTiOnS}

In this section, we show in fact that there exists for every $p \in \mathbb{R}^{n}$ a positive constant $c_{\varepsilon}(p)$ such that solutions to (1) starting from hyperplanes $z=p \cdot x$ remain at a small distance from hyperplanes with the same normal and moving with (uniformly bounded in $\varepsilon$ ) speed $c_{\varepsilon}(p) \varepsilon^{\alpha-1}$. Moreover we study the limit as $\varepsilon \rightarrow 0$ to $c_{\varepsilon}(p) \varepsilon^{\alpha-1}$ : this will be the average speed of hyperplanes.

Theorem 2.1. Let $v^{\varepsilon}$ be the solution to (4). Then there exists a unique $c_{\varepsilon}(p)>0$ such that

$$
\left|v^{\varepsilon}(x, t)-p \cdot x-c_{\varepsilon}(p) t\right| \leq K
$$

where $K$ depends on the Lipschitz norm of $g$. Moreover

$$
0<c_{\varepsilon}(p) \leq \varepsilon^{1-\alpha}\|g\|_{\infty} .
$$

The proof of this theorem is based on the a priori estimates provided in Theorem 1.1, and follows as in [9, Thm 3.1] (see also [6] for a proof of a more general result of this kind).

Remark 1. Observe that if $u^{\varepsilon}$ is the solution to (1) with initial datum $u^{\varepsilon}(x, 0)=p \cdot x$, then $v^{\varepsilon}(x, t)=$ $\frac{1}{\varepsilon} u^{\varepsilon}\left(\varepsilon x, \varepsilon^{2-\alpha} t\right)$ is the solution to (4). So Theorem 2.1 implies

$$
\left|u^{\varepsilon}(x, t)-p \cdot x-c^{\varepsilon}(p) \varepsilon^{\alpha-1} t\right| \leq K \varepsilon .
$$

Remark 2. Note that, when $\alpha=1$, the speed $c^{\varepsilon}(p)$ does not depend on $\varepsilon$. When $\alpha=1$ and the Laplacian in (1) is substituted by the mean curvature operator, the existence of traveling wave solutions has been established in [9], under suitable assumptions on the forcing term $g$.

Proposition 2.2. Let $c^{\varepsilon}(p)$ as in Theorem 2.1 and assume that $g=g(u): \mathbb{R} \rightarrow \mathbb{R}$. Then

$$
\lim _{\varepsilon \rightarrow 0} c^{\varepsilon}(p) \varepsilon^{\alpha-1}=c(p)= \begin{cases}-\int_{0}^{1} g(s) d s & p \neq 0 \\ -\left(\int_{0}^{1} \frac{1}{g(s)} d s\right)^{-1} & p=0, g<0 \\ 0 & p=0, \max g=0 .\end{cases}
$$


Proof. For $p=0$, equation (4) becomes

$$
\left\{\begin{array}{l}
v^{\prime}(t)+\varepsilon^{1-\alpha} g(v)=0 \\
v(0)=0
\end{array}\right.
$$

So, if $g<0$, by direct integration we get

$$
\int_{0}^{v(t)} \frac{d s}{g(s)}=-\varepsilon^{1-\alpha} t
$$

In particular $v$ is monotone increasing, unbounded and

$$
\lim _{t \rightarrow+\infty} \frac{v(t)}{t}=-\varepsilon^{1-\alpha}\left(\int_{0}^{1} \frac{1}{g(s)} d s\right)^{-1}=c^{\varepsilon}(0) .
$$

If, on the other hand there exists $v_{0} \in[0,1]$ such that $g\left(v_{0}\right)=0$, then by comparison $v_{0}-1 \leq v(t) \leq v_{0}$ for every $t$, and then $c^{\varepsilon}(0)=0$.

For $p \neq 0$, we define $\chi_{p}: \mathbb{R} \times(0,+\infty) \rightarrow \mathbb{R}$ as $\chi_{p}(p \cdot x, t)=w^{\varepsilon}(x, t)$, where $w^{\varepsilon}$ solves $(5)$. Then $\chi_{p}$ solves

$$
\left\{\begin{array}{l}
\left(\chi_{p}\right)_{t}-|p|^{2}\left(\chi_{p}\right)_{z z}+\varepsilon^{1-\alpha} g\left(\chi_{p}(z, t)+z\right)=0 \quad t>0, z \in \mathbb{R} \\
\chi_{p}(z, 0)=0
\end{array}\right.
$$

and is 1-periodic. We integrate in space and time (14) and, using periodicity of $\chi_{p}$, we obtain

So

$$
\int_{0}^{1} \frac{1}{t} \int_{0}^{t}\left(\chi_{p}\right)_{t} d t d z=-\varepsilon^{1-\alpha} \frac{1}{t} \int_{0}^{t} \int_{0}^{1} g\left(\chi_{p}+z\right) d z d t
$$

$$
\frac{1}{t} \int_{0}^{t}\left(\chi_{p}\right)_{t} d t=\frac{\chi_{p}(z, t)}{t} \rightarrow c^{\varepsilon}(p) \quad t \rightarrow+\infty .
$$

Using the a priori bounds on the derivative $\left(\chi_{p}\right)_{z}$ given by Theorem 1.1 and the fact that $g$ is Lipschitz, we get

$$
\int_{0}^{1} g(z+\chi(z, t)) d z=\int_{0}^{1} g\left(z+\int_{0}^{1} \chi(\zeta, t) d \zeta\right) d z+O\left(\varepsilon^{\frac{1-\alpha}{4}}\right)=\int_{0}^{1} g(z) d z+O\left(\varepsilon^{\frac{1-\alpha}{4}}\right)
$$

where the error term $O\left(\varepsilon^{\frac{1-\alpha}{4}}\right)$ is independent of $t$. This gives

$$
c^{\varepsilon}(p)=-\varepsilon^{1-\alpha} \int_{0}^{1} g(z) d z+o\left(\varepsilon^{1-\alpha}\right) .
$$

\section{Asymptotic speed of PROpagation}

In this section we provide the asymptotic speed of propagation of solutions to (1) as $\varepsilon \rightarrow 0$ starting from almost planar initial datum. When the initial datum is an hyperplane, we obtain the homogenization limit of solutions to (4).

Theorem 3.1. Let $u_{\varepsilon}$ be the solution to

$$
\left\{\begin{array}{l}
\frac{\partial u^{\varepsilon}}{\partial t}-\varepsilon^{\alpha} \Delta u^{\varepsilon}+g\left(\frac{u^{\varepsilon}}{\varepsilon}\right)=0 \quad t>0, x \in \mathbb{R}^{n} \\
u^{\varepsilon}(x, 0)=u_{0}(x)
\end{array}\right.
$$


Assume that there exists $p \in \mathbb{R}^{n}$ such that

$$
u_{0}(x)-p \cdot x=v_{0}(x) \in L^{\infty}\left(\mathbb{R}^{n}\right) .
$$

Then

$$
p \cdot x+c(p) t+\inf v_{0} \leq \lim \inf _{\varepsilon \rightarrow 0} u^{\varepsilon}(x, t) \leq \lim \sup _{\varepsilon \rightarrow 0} u^{\varepsilon}(x, t) \leq p \cdot x+c(p) t+\sup v_{0}
$$

where $c(p)$ is defined in (13).

In particular, if $u_{0}(x)-p \cdot x \equiv k$ for $k \in \mathbb{R}$, then the solution $u^{\varepsilon}$ to (15) satisfies

$$
\lim _{\varepsilon \rightarrow 0} u_{\varepsilon}(x, t)=p \cdot x+c(p) t+k \quad \text { uniformly in } x \in \mathbb{R}^{n}, t \geq 0 .
$$

Proof. We consider the solutions $u_{+}^{\varepsilon}$, $u_{-}^{\varepsilon}$ to (15) with initial datum $u_{+}^{\varepsilon}(x, 0)=p \cdot x+\varepsilon\left[\frac{\sup v_{0}}{\varepsilon}\right]+\varepsilon$ and $u_{-}^{\varepsilon}(x, 0)=p \cdot x+\varepsilon\left[\frac{\inf v_{0}}{\varepsilon}\right]$. By comparison $u_{-}^{\varepsilon}(x, t) \leq u^{\varepsilon}(x, t) \leq u_{+}^{\varepsilon}(x, t)$ for every $x, t$. We can rewrite

$$
u_{-}^{\varepsilon}(x, t)=p \cdot x+\varepsilon w^{\varepsilon}\left(\frac{x}{\varepsilon}, \frac{t}{\varepsilon^{2-\alpha}}\right)+\varepsilon\left[\frac{\inf v_{0}}{\varepsilon}\right]
$$

and analogously $u_{+}^{\varepsilon}$, where $w^{\varepsilon}$ is the solution to (5).

So we get that

$$
u_{-}^{\varepsilon}(x, t)=p \cdot x+\varepsilon^{\alpha-1} c^{\varepsilon}(p) t+\varepsilon\left(w^{\varepsilon}\left(\frac{x}{\varepsilon}, \frac{t}{\varepsilon^{2-\alpha}}\right)-c^{\varepsilon}(p) \frac{t}{\varepsilon^{2-\alpha}}\right)+\varepsilon\left[\frac{\inf v_{0}}{\varepsilon}\right]
$$

and analogously for $u_{+}^{\varepsilon}$. By comparison we get that

$$
\begin{aligned}
\varepsilon\left(w^{\varepsilon}\left(\frac{x}{\varepsilon}, \frac{t}{\varepsilon^{2-\alpha}}\right)-c^{\varepsilon}(p) \frac{t}{\varepsilon^{2-\alpha}}\right)+\varepsilon\left[\frac{\inf v_{0}}{\varepsilon}\right] & \leq u^{\varepsilon}(x, t)-p \cdot x-\varepsilon^{\alpha-1} c^{\varepsilon}(p) t \\
& \leq \varepsilon\left(w^{\varepsilon}\left(\frac{x}{\varepsilon}, \frac{t}{\varepsilon^{2-\alpha}}\right)-c^{\varepsilon}(p) \frac{t}{\varepsilon^{2-\alpha}}\right)+\varepsilon\left[\frac{\sup v_{0}}{\varepsilon}\right]+\varepsilon
\end{aligned}
$$

Recalling Theorem 2.1, and letting $\varepsilon \rightarrow 0$, we get the thesis.

\section{REFERENCES}

[1] N. Alibaud, A. Briani, R. Monneau. Diffusion as a singular homogenization of the Frenkel-Kontorova model. J. Differential Equations 251, no. 4-5, 785-815, 2011.

[2] G. Barles, P. E. Souganidis. Space-time periodic solutions and long-time behavior of solutions to quasi-linear parabolic equations. SIAM J. Math. Anal. 32, no. 6, 1311-1323, 2011.

[3] H. Berestycki, F. Hamel. Generalized travelling waves for reaction-diffusion equations. In: Perspectives in Nonlinear Partial Differential Equations. In honor of H. Brezis. Contemp. Math. 446, Amer. Math. Soc., 101-123, 2007.

[4] L.A. Caffarelli, R. Monneau. Counter-example in three dimension and homogenization of geometric motions in two dimension. Arch. Ration. Mech. Anal. 212, no. 2, 503-574, 2014.

[5] P. Cardaliaguet, P.-L. Lions, P. E. Souganidis. A discussion about the homogenization of moving interfaces. J. Math. Pures Appl. 91, 339-363, 2009.

[6] A. Cesaroni, N. Dirr, F. Dragoni, M. Novaga. Homogenization of a semilinear heat equation. Preprint, 2016.

[7] A. Cesaroni, M. Novaga. Long-time behavior of the mean curvature flow with periodic forcing. Comm. Partial Differential Equations 38, 780-801, 2013.

[8] A. Cesaroni, M. Novaga, E. Valdinoci. Curve shortening flow in heterogeneous media. Interfaces and Free Boundaries 13(4), 617-635, 2011.

[9] N. Dirr, G. Karali, N. K. Yip. Pulsating wave for mean curvature flow in inhomogeneous medium. Eur. J. of Applied Mathematics 19, 661-699, 2008. 
[10] N. Dirr, N. K. Yip. Pinning and de-pinning phenomena in front propagation in heterogeneous media. Interfaces and Free Boundaries 8(1), 79-109, 2006.

[11] N. Dirr, P.E. Souganidis. Large-time behavior for viscous and nonviscous Hamilton-Jacobi equations forced by additive noise. SIAM J. Math. Anal. 37, no. 3, 777-796, 2005.

[12] R. Jerrard, Singular limits of scalar Ginzburg-Landau equations with multiple-well potentials, Adv. Differential Equations 2 (1) $1-38,1997$

[13] P.-L. Lions, Quelques remarques sur les problémes elliptiques quasilinéaires du second ordre, J. Analyse Math. 45, 234-254, 1985.

[14] P.-L. Lions, P. E. Souganidis. Homogenization of degenerate second-order PDE in periodic and almost periodic environments and applications. Ann. Inst. H. Poincaré Anal. Non Linéaire 22, no. 5, 667-677, 2005.

[15] G. Namah, J.-M. Roquejoffre. Convergence to periodic fronts in a class of semi-linear parabolic equations. Nonlinear Diff. Eq. Appl. 4, 521-536,1998.

[16] M. H. Protter, H. F. Weinberger. Maximum principles in differential equations. Springer-Verlag, New York, 1984 